\title{
Interference-Limited Opportunistic Relaying with Reactive Sensing
}

\author{
Aggelos Bletsas, Member, IEEE, Antonis G. Dimitriou, Member, IEEE, \\ and John N. Sahalos, Fellow, IEEE
}

\begin{abstract}
This work evaluates opportunistic relaying in the presence of thermal noise as well as interference, when channel sensing is conducted reactively, in slow fading environments. The studied scenario employs a single gateway that provides access towards several destinations with weak links and exploits a network of intermediate relays. In sharp contrast to prior art, no inter-relay channel state information or communication is assumed, no network coding is needed, while low-complexity receivers at each destination are employed.

It is shown that information can be relayed without delay, while harvesting benefits of cooperative diversity, even at the presence of interference. The participating relays are required to offer strong paths towards source and destination, while at the same time they are as "isolated" as possible from each other. From that perspective, the notion of relay "usefulness" is redefined in both noise and interference-limited environments, under opportunistic relaying.
\end{abstract}

Index Terms-Multiple access, fading, wireless networks.

\section{INTRODUCTION}

$\mathbf{S}$ IGNIFICANT progress has been made in cooperative relaying, especially in thermal noise-limited environments [1]. It was recently shown that cooperative relays, distributed in thermal noise-limited environments, are useful even when they do not transmit but instead, they cooperatively listen, giving priority to single transmission of an opportunistically selected relay [2]. Alternatively, the source could transmit messages towards all destinations, and relays delay retransmissions, until messages for all destinations have been transmitted. Communication towards a given destination is completed after relays network-encode and broadcast a combined message for all destinations. This is the approach of network coding (e.g. see [3] and references therein). Thus, both approaches above trade delay for increased reliability with the help of cooperative relays in noise-limited environments.

In this work, we evaluate opportunistic relaying with reactive channel sensing, in both noise and interference-limited, slow fading environments, when access from a central gateway towards various destinations is problematic (i.e. the associated channel links are weak) and intermediate relays are necessary.

Manuscript submitted August 8, 2008; revised April 11, 2009 and September 7, 2009; accepted September 8, 2009. The associate editor coordinating the review of this letter and approving it for publication was S. Affes.

A. Bletsas was with the Radio Communications Laboratory, and is now with the ECE Dept., Technical Univ. of Crete (e-mail: aggelos@telecom.tuc.gr).

A. Dimitriou is with the ECE Dept., Aristotle Univ. of Thessaloniki (e-mail: antodimi@mri.ee.auth.gr).

J. N. Sahalos is with the Radio Communications Laboratory, Dept. of Physics, Aristotle Univ. of Thessaloniki (e-mail: sahalos@auth.gr).

Work was implemented in the context of the Telecommunications Platform of Innovation Pole of C.M. Greece, through the O.P. Competitiveness 3rd Community Support Program, and was funded from the Hellenic StateMinistry of Development-General Secretariat for Research and Technology.

Digital Object Identifier 10.1109/TWC.2010.01.081128

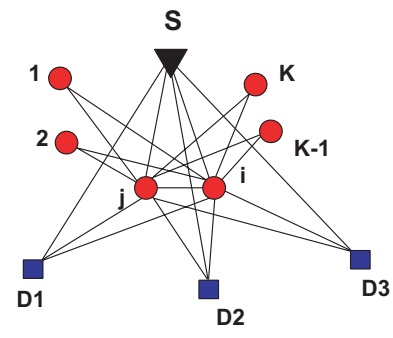

Fig. 1. The connectivity setup of the studied scenario: source $s$ acts as an access point (gateway) and transmits towards two or more distinct destinations, through $K \geq 2$ relays. The special case of three destinations $(D 1, D 2, D 3)$ is depicted. Direct links from $s$ to destinations are assumed week, necessitating the utilization of relays.

In sharp contrast to prior art, no inter-relay channel state information or inter-relay communication is assumed, no network coding is employed, while low-complexity receivers at each destination are utilized. This work could be of potential value in dense, low-mobility wireless scenarios, as in modern urban environments. The latter could offer relay densities on the order of 1000 terminals $/ \mathrm{km}^{2}$, according to recent measurements [4].

Sections II and III describe system and protocol model, respectively. Section IV provides the analysis for reactive channel sensing and relay selection, section $\mathrm{V}$ briefly discusses alterative channel sensing and relay selection techniques and section VI provides the numerical results. Finally, Section VII provides the conclusion.

\section{SyStem ModeL}

If node " $\mathrm{A}$ " transmits to node "B", while an interfering node " $\mathrm{C}$ " is transmitting to a different destination, then the received signal at node " $\mathrm{B}$ " is denoted as:

$$
y_{\mathrm{B}}=\alpha_{\mathrm{AB}} x_{\mathrm{A}}+\alpha_{\mathrm{CB}} x_{\mathrm{C}}+n_{\mathrm{B}},
$$

where $x_{\mathrm{A}}, x_{\mathrm{C}}$ are the signals transmitted from node $\mathrm{A}, \mathrm{C}$ respectively, $\alpha_{\mathrm{AB}}, \alpha_{\mathrm{CB}}$ are the complex channel gains for the link $\mathrm{A} \rightarrow \mathrm{B}, \mathrm{C} \rightarrow \mathrm{B}$ respectively, and $n_{\mathrm{B}} \sim \mathcal{C N}\left(0, N_{0}\right)$ is the complex, circularly symmetric additive white Gaussian noise (AWGN) at node B, with zero mean and variance $N_{0}$. Operator $\mathbb{E}\{x\}$ denotes the expected value of random variable $x$. For each link, let $\gamma_{\mathrm{AB}} \triangleq\left|\alpha_{\mathrm{AB}}\right|^{2}$ be the instantaneous squared channel strength, which obeys an exponential distribution with parameter $\lambda_{\mathrm{AB}}=1 / \mathbb{E}\left\{\gamma_{\mathrm{AB}}\right\}$, corresponding to Rayleigh fading. Notation []$^{*},[]^{T},[]^{\dagger}$ further denotes the conjugate, transpose and conjugate transpose operator, respectively. Finally, diag $\left[\begin{array}{ll}x & y\end{array}\right]$ denotes the two-by-two diagonal matrix 


\section{Epoch $\mathbf{E} \quad$ Epoch $\mathbf{n + 1}$}

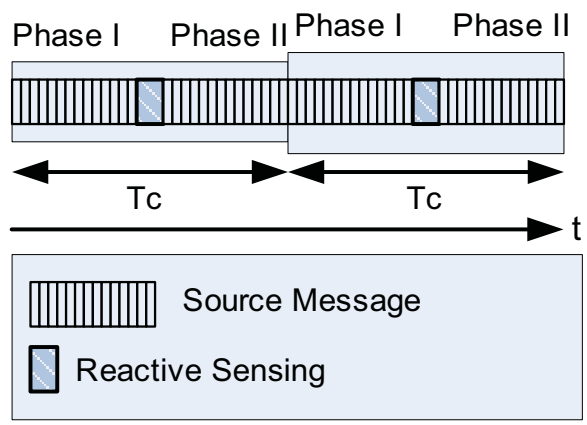

Fig. 2. Each epoch spans a duration equal to the channel coherence time. Two consecutive messages (phase I and phase II) are transmitted from the source towards two different destinations. Reactive sensing and opportunistic relay selection is performed for each phase I source message.

with $\left[\begin{array}{ll}x & y\end{array}\right]$ at each main diagonal, and $\mathcal{I}_{2}$ denotes the two-bytwo identity matrix.

\section{PROTOCOL}

The studied scenario employs a single gateway that provides access towards two or more destinations with weak links and exploits a network of $K \geq 2$ intermediate, regenerative relays. All involved radios are assumed half-duplex. Fig. 1 depicts the connectivity setup for the special case of three destinations. We denote the set of relays as $\mathcal{S}_{\mathrm{R}}=\{1,2, \ldots, K\}$ and further denote $\mathcal{S}_{\mathrm{R}}^{i}=\mathcal{S}_{\mathrm{R}}-\{i\}, i \in \mathcal{S}_{\mathrm{R}}$, the set of relays that does not include relay $i$ (length $\left|\mathcal{S}_{\mathrm{R}}^{i}\right|=K-1$ ).

The channel is assumed constant for a duration of $T_{c}$ (channel coherence time), which spans one epoch and changes independently at the next epoch (quasi-static, flat fading). We are particularly interested in slow-fading wireless environments, where $T_{c}$ is on the order of hundreds of milliseconds (as in slow mobility scenarios or community rooftop networks). At each epoch of the protocol, there are exactly two different messages transmitted by the source: phase I message, which is assisted by opportunistic relaying and phase II message, assisted by conventional regenerative relaying (Fig. 2).

During phase I, the source transmits towards a new destination while relay $i$ forwards different information to a different destination without delay i.e. without waiting the source to complete its transmission, as in strictly noise-limited setups. During phase II, an opportunistically selected relay $b \neq i$ forwards to the appropriate destination while source transmits a message for a new destination. Operation is similar in the next epoch; the opportunistically selected relay at epoch $n$, becomes the interfering relay $i$ at the consecutive epoch $n+1$ (Fig. 3).

The selected ("best") relay $b$ is chosen at the end of phase I opportunistically, among the relays that have successfully received the message, in this both noise and interferencelimited environment:

$$
\gamma_{b d}=\max \left\{\gamma_{k d}\right\}, k \in \mathcal{D}_{\ell} \subseteq \mathcal{S}_{\mathrm{R}}^{i},
$$

where the subset $\mathcal{D}_{\ell} \subseteq \mathcal{S}_{\mathrm{R}}^{i}$ includes all relays (excluding relay $i$ ) that have successfully received the message at the end of phase I (i.e. $\left|\mathcal{D}_{\ell}\right| \geq 1$ ).
Such reactive relay selection (i.e. selection in response to source transmission) can be performed in a distributed fashion, without network channel state information (CSI) knowledge at each relay or any centralized network controller. One possible approach is the method of distributed timers proposed in [5], with each relay in $\mathcal{D}_{\ell} \subseteq \mathcal{S}_{\mathrm{R}}^{i}$ estimating its own instantaneous channel link $\gamma_{k d}$ towards destination. This can be accomplished by listening a pilot signal from destination. Then the participating relays contend for carrier-sense-multiple access (CSMA), where the backoff timer of each relay is initialized with a value that depends on its own instantaneous signal strength $\gamma_{k d}$. The timer of the relay with the strongest path towards destination expires first and the "best" relay of eq. (2) acquires the medium. Relay selection could be completed within a fraction of channel coherence time, even though no relay (or any controller in the network) has measured channel conditions of the interfering relay or other relays towards destination. Statistical analysis of distributed relay selection as above has been reported in [5].

We further denote source transmission power as $\mathcal{P}_{\text {source }}$, relay transmission power as $\mathcal{P}$ relay and total transmission power $\mathcal{P}=\mathcal{P}_{\text {source }}+\mathcal{P}_{\text {relay }}$. Assuming $\zeta \in(0,1)$, then $\mathcal{P}_{\text {source }}=\zeta \mathcal{P}$ and $\mathcal{P}_{\text {relay }}=(1-\zeta) \mathcal{P}$. The power allocation parameter $\zeta$ is assumed constant across time. Its optimization would require global CSI across all involved links in the network, which is a strong assumption not made in this work. Finally, we denote transmit signal-to-noise ratio $S N R=\mathcal{P} / N_{0}$.

For each "phase I message" transmitted by the source, the received signal at the final destination can be denoted as a two-by-one vector $\mathbf{y}$, as it consists of two time-separated parts (Fig. 3): the first part is received during phase I, when the source transmits the signal of interest (SOI) and an interfering relay transmits a different symbol; the second part is transmitted during phase II, when the source transmits towards a different destination and the "best" relay retransmits, assuming that a best relay exists $\left(\left|\mathcal{D}_{\ell}\right| \geq 1\right)$. For $\left|\mathcal{D}_{\ell}\right| \geq 1$ :

$$
\begin{gathered}
\mathbf{y}=\sqrt{\mathcal{P}} \underbrace{\left[\begin{array}{c}
\sqrt{\zeta} \alpha_{s d} \\
\sqrt{(1-\zeta)} \alpha_{b d}
\end{array}\right]}_{\mathbf{a}} x_{0}+\sqrt{\mathcal{P}} \underbrace{\left[\begin{array}{c}
\sqrt{1-\zeta} \alpha_{i d} \\
0
\end{array}\right]}_{\mathbf{c}_{\mathbf{1}}} x_{1}+ \\
+\sqrt{\mathcal{P}} \underbrace{\left[\begin{array}{c}
0 \\
\sqrt{\zeta} \alpha_{s d}
\end{array}\right]}_{\mathbf{c}_{2}} x_{2}+\mathbf{n} \Rightarrow
\end{gathered}
$$

$$
\mathbf{y}=\sqrt{\mathcal{P}} \mathbf{a} x_{0}+\underbrace{\sqrt{\mathcal{P}} \sum_{i=1}^{2} \mathbf{c}_{\mathbf{i}} x_{i}+\mathbf{n}}_{\mathbf{v}}=\sqrt{\mathcal{P}} \mathbf{a} x_{0}+\mathbf{v},
$$

where $x_{0}$ is the SOI and $x_{1}, x_{2}$ the interfering signals, with $\mathbb{E}\left\{\mathbf{n}^{*} \mathbf{n}^{T}\right\}=N_{0} \mathcal{I}_{2}$ and $\mathbb{E}\left\{\left|x_{0}\right|^{2}\right\}=\mathbb{E}\left\{\left|x_{1}\right|^{2}\right\}=$ $\mathbb{E}\left\{\left|x_{2}\right|^{2}\right\}=1$ (e.g. PSK modulation). For $\left|\mathcal{D}_{\ell}\right|=0$, there is no best relay transmission and thus, $\mathbf{a}=\left[\begin{array}{lll}\sqrt{\zeta} \alpha_{s d} & 0\end{array}\right]^{T}$ in eq. (4).

From eqs. (3), (4) it is shown that there are two interferers of unequal power $\left(\mathbf{c}_{1}, \mathbf{c}_{2}\right)$, while there is strong correlation between the compound channel a of SOI $x_{0}$ and the compound channel $\mathbf{c}_{2}$ of the interfering signal $x_{2}$. That is due to common 


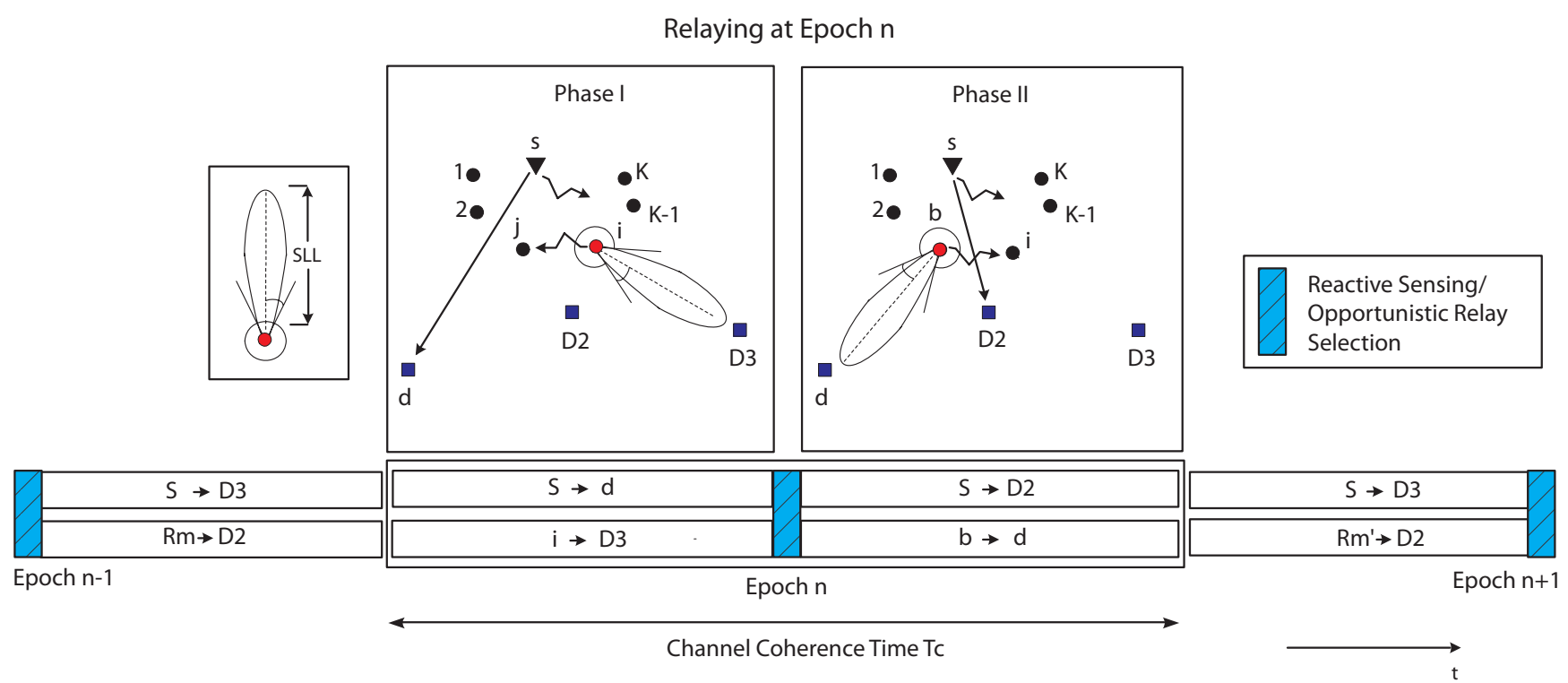

Fig. 3. Phase I: the source transmits towards destination $d$ while relay $i$ (among $K$ relays) forwards information to a different destination. Phase II: an opportunistically selected relay $b \neq i$ forwards to the appropriate destination while source transmits a message for a new destination. The two phases constitute an epoch.

random variable $\alpha_{s d}$, which remains constant during each epoch, as already described. It is noted that prior art has mainly studied channel correlation among the elements of a (which is relevant to SOI) or correlation among the elements of $\mathbf{v}$ (which is relevant to the interfering signals) (e.g. [6],[7]), but not among $\mathbf{a}$ and $\mathbf{v}$, as in this work, to the best of our knowledge.

The receiver at each destination linearly processes the received vector with $\mathbf{w}^{*} \mathbf{y}$, where $\mathbf{w}$ depends on the specified receiver. The simplest case is the selection diversity receiver that does not optimally combine the two signal parts, received during phase I and II. Instead, the receiver operates independently on the two messages and succeeds if either reception, during phase I or II succeeds. An alternative approach employs optimum combining, at the expense of additional CSI requirements. The main focus is on selection diversity receiver (SR), even though optimum combining receiver is also discussed (OC). Analysis of the above, interference-limited, opportunistic relaying (IOR) approach is presented in this work, employing outage analysis, i.e. probability evaluation of the event that target rate is greater than what can be supported from the wireless channel.

\section{Analysis of IOR with Reactive Sensing}

At each epoch of the protocol, there are exactly two different messages transmitted by the source: phase I message, which is assisted by opportunistic relaying and phase II message, assisted by conventional regenerative relaying (Fig. 2). Performance of conventional, non-OR phase II message can be trivially calculated and we focus on the OR case. During phase I of epoch $n$ (Fig. 2), there are at most two transmitting nodes (Fig. 3): the source and the opportunistically selected relay, which both transmit towards different destinations. Given the half-duplex constraint, the opportunistically selected relay $b$ at epoch $n$ cannot be the same as the forwarding (interfering) relay $i$ during the (immediately) previous epoch $n-1$ (Figs. 2, 3). The destination node at epoch $n$ is denoted as $d$ and the interfering relay as $i(b \neq i)$. The interfering relay $i$ was the opportunistically selected relay during epoch $n-1$, according to the (different) channel conditions of that epoch.

Next, we denote by $\{$ out $\mid I=i\}$ the outage event at the presence of interfering relay $i$ and by $\{$ out $\mid I=$ null $\}$ the outage event at the absence of an interfering relay. The unconditional outage probability, for a given destination, at a given epoch becomes:

$$
\begin{aligned}
& \operatorname{Pr}\{\text { out }\}=\operatorname{Pr}\{\text { out } \mid I=\text { null }\} \operatorname{Pr}\{I=\text { null }\}+ \\
& \quad+\sum_{i=1}^{K} \operatorname{Pr}\{\text { out } \mid I=i\} \operatorname{Pr}\{I=i\} \Rightarrow \\
& \operatorname{Pr}\{\text { out }\} \simeq \sum_{i=1}^{K} \operatorname{Pr}\{\text { out } \mid I=i\} \operatorname{Pr}\{I=i\} .
\end{aligned}
$$

The above approximation is valid because at the absence of interference, outage performance is strictly thermal-noiselimited (event $\{$ out $I=$ null $\}$ ), while at the presence of an interfering relay $i$ (event $\{$ out $\mid I=i\}$ ), performance is both thermal-noise as well as interference-limited; thus, $\operatorname{Pr}\{$ out $\mid I=$ null $\}<<\operatorname{Pr}\{$ out $\mid I=i\}, \forall i \in \mathcal{S}_{\mathrm{R}}$. Additionally, the event of 'zero number of successful decoding during epoch $n-1$ ' (and thus, $I=$ null at epoch $n$ ), becomes less likely for large number of relays, implying that $\operatorname{Pr}\{I=$ null $\}$ decreases with increasing number of relays. Numerical results of section VI demonstrate that performance is dominated by interference and not thermal noise, further validating the above.

We remark that the prior probabilities $\operatorname{Pr}\{I=i\}, \forall i \in \mathcal{S}_{\mathrm{R}}$, for a given epoch $n$, depend on the selected relay during the previous epoch $n-1$, which in turn, depends on the selected relay during epoch $n-2$ and so forth. Therefore, prior probability $\operatorname{Pr}\{I=i\}, \forall i \in \mathcal{S}_{\mathrm{R}}$ depends on epoch 
number. However, we observe from Eq. (5) that

$$
\begin{aligned}
& \operatorname{Pr}\{\text { out }\} \leq \max _{i \in \mathcal{S}_{\mathrm{R}}}\{\operatorname{Pr}\{\text { out } \mid I=i\}\}, \\
& \operatorname{Pr}\{\text { out }\} \geq \min _{j \in \mathcal{S}_{\mathrm{R}}}\{\operatorname{Pr}\{\text { out } \mid I=j\}\} .
\end{aligned}
$$

Therefore, the upper and lower bounds calculated above evaluate outage performance without any dependence on epoch number.

Conditioned on interfering relay $i$ for a given epoch, the outage probability is given by:

$$
\begin{aligned}
& \operatorname{Pr}\{\text { out } \mid \text { Interfering Relay }=\text { Relay } i\} \triangleq \operatorname{Pr}\{\text { out } \mid I=i\} \\
& =\sum_{\mathcal{D}_{\ell}} \operatorname{Pr}\left\{\text { out } \mid \mathcal{D}_{\ell}, I=i\right\} \operatorname{Pr}\left(\mathcal{D}_{\ell} \mid I=i\right) \\
& =\operatorname{Pr}\left\{\text { out }|I=i,| \mathcal{D}_{\ell} \mid=0\right\} \operatorname{Pr}\left(\left|\mathcal{D}_{\ell}\right|=0 \mid I=i\right)+ \\
& +\sum_{\substack{\mathcal{D}_{\ell} \\
\left|\mathcal{D}_{\ell}\right| \geq 1}} \operatorname{Pr}\left\{\text { out }\left|\mathcal{D}_{\ell},\right| \mathcal{D}_{\ell} \mid \geq 1, I=i\right\} \operatorname{Pr}\left(\mathcal{D}_{\ell} \mid I=i\right),
\end{aligned}
$$

where the term $\operatorname{Pr}\left\{\right.$ out $\left.|I=i,| \mathcal{D}_{\ell} \mid=0\right\}$ denotes the case where no relay successfully decodes the message, and thus, destination reception is solely relied on direct communication. Consecutively,

$$
\begin{aligned}
& \operatorname{Pr}\left\{\text { out }|I=i,| \mathcal{D}_{\ell} \mid=0\right\}= \\
& =\operatorname{Pr}\left\{\frac{1}{2} \log _{2}\left(1+\frac{\zeta \mathcal{P} \gamma_{s d}}{(1-\zeta) \mathcal{P} \gamma_{i d}+N_{0}}\right)<R\right\} \\
= & \operatorname{Pr}\left(\frac{\zeta \mathcal{P} \gamma_{s d}}{(1-\zeta) \mathcal{P} \gamma_{i d}+N_{0}}<\Theta\right)= \\
= & 1-e^{-\lambda_{s d} \frac{\Theta N_{0}}{\zeta P}}\left[1+\Theta \frac{1-\zeta}{\zeta} \frac{\lambda_{s d}}{\lambda_{i d}}\right]^{-1}, \Theta=2^{2 R}-1,
\end{aligned}
$$

where $R$ is the target rate in $\mathrm{bps} / \mathrm{Hz}$ and we have exploited independence between $\gamma_{i d}$ and $\gamma_{s d}$. It is noted that the statistics of all channel gains, related to the interfering relay $i$ at a given epoch $n$, are not affected by the selection process of that relay during the previous epoch $n-1$; that is due to the fact that the channel has changed during epoch $n$ (see the duration of channel coherence time $T_{c}$ in Figs. 2, 3). Thus,

$$
\begin{aligned}
& \operatorname{Pr}\left(\mathcal{D}_{\ell} \mid I=i\right)=\prod_{k \in \mathcal{D}_{\ell}} \operatorname{Pr}\left\{\frac{\zeta \mathcal{P} \gamma_{s k}}{(1-\zeta) \mathcal{P} \gamma_{i k}+N_{0}}>\Theta\right\} \times \\
& \times \prod_{m \notin \mathcal{D}_{\ell}} \operatorname{Pr}\left\{\frac{\zeta \mathcal{P} \gamma_{s m}}{(1-\zeta) \mathcal{P} \gamma_{i m}+N_{0}}<\Theta\right\} \\
& =\prod_{k \in \mathcal{D}_{\ell}} e^{-\frac{\lambda_{s k} \Theta N_{0}}{\zeta \mathcal{P}}}\left[1+\Theta \frac{1-\zeta}{\zeta} \frac{\lambda_{s k}}{\lambda_{i k}}\right]^{-1} \times \\
& \quad \times \prod_{m \notin \mathcal{D}_{\ell}}\left(1-e^{-\frac{\lambda_{s m} \Theta N_{0}}{\zeta \mathcal{P}}}\left[1+\Theta \frac{1-\zeta}{\zeta} \frac{\lambda_{s m}}{\lambda_{i m}}\right]^{-1}\right) .
\end{aligned}
$$

In the above analysis, the only missing element is probability $\operatorname{Pr}\left\{\right.$ out $\left.\left|\mathcal{D}_{\ell},\right| \mathcal{D}_{\ell} \mid \geq 1, I=i\right\}$ of Eq. (7). That probability depends on the specifics of the receiver at each destination and is calculated in the following subsections.

\section{A. Optimum Combining (OC) Receiver}

For optimum combining, the weighting vector $\mathbf{w}_{\mathrm{OC}}$ that maximizes the signal-to-interference-and-noise ratio (SINR) is proportional to $\mathbf{R}_{\mathbf{v}}^{-\mathbf{1}}$ a, with a defined in section III and $\mathbf{R}_{\mathbf{v}}$ given by:

$$
\mathbf{R}_{\mathbf{v}}=\mathbb{E}\left\{\mathbf{v}^{*} \mathbf{v}^{T}\right\}=N_{0} \mathcal{I}_{2}+\mathcal{P} \sum_{i=1}^{2} \mathbf{c}_{i}^{*} \mathbf{c}_{i}^{T} .
$$

Setting $\mathbf{w}_{\text {OC }}=\mathbf{R}_{\mathbf{v}}^{-\mathbf{1}}$ a, the received SINR at the destination is simplified to

$$
\gamma_{\mathrm{OC}} \triangleq \mathcal{P} \frac{\mathbf{w}^{\dagger} \mathbf{a} \mathbf{a}^{\dagger} \mathbf{w}}{\mathbf{w}^{\dagger} \mathbf{R}_{\mathbf{v}} \mathbf{w}}=\mathbf{a}^{\dagger} \mathbf{R}_{\mathbf{v} \mathbf{0}}^{-\mathbf{a}}, \quad \mathbf{R}_{\mathrm{v} \mathbf{0}}=\frac{1}{\mathcal{P}} \mathbf{R}_{\mathbf{v}},
$$

and consecutively,

$$
\begin{aligned}
& \operatorname{Pr}\left\{\text { out }\left|\mathcal{D}_{\ell},\right| \mathcal{D}_{\ell} \mid \geq 1, I=i\right\}_{\mathrm{OC}} \triangleq \operatorname{Pr}\left(\gamma_{\mathrm{oc}}<\Theta\right) \\
& =\operatorname{Pr}\left(\frac{\zeta \mathcal{P} \gamma_{s d}}{(1-\zeta) \mathcal{P} \gamma_{i d}+N_{0}}+\frac{(1-\zeta) \mathcal{P} \gamma_{b d}}{\zeta \mathcal{P} \gamma_{s d}+N_{0}}<\Theta\right) \\
& \leq \operatorname{Pr}\left(\frac{\zeta \mathcal{P} \gamma_{s d}}{(1-\zeta) \mathcal{P} \gamma_{i d}+N_{0}}<\Theta \bigcap \frac{(1-\zeta) \mathcal{P} \gamma_{b d}}{\zeta \mathcal{P} \gamma_{s d}+N_{0}}<\Theta\right)= \\
& =\operatorname{Pr}\left\{\text { out }\left|\mathcal{D}_{\ell},\right| \mathcal{D}_{\ell} \mid \geq 1, I=i\right\}_{\mathrm{SR}} .
\end{aligned}
$$

The above bound corresponds to the performance of the selection receiver calculated immediately below. It is remarked that $\mathbf{w}_{\mathrm{OC}}$ (and consecutively, optimum combining) requires CSI knowledge regarding the interfering terminal $\left(\alpha_{i d}\right)$, in contrast to the selection receiver. Thus, the improved performance of optimum combining (compared to selection receiver) is achieved at the expense of additional required channel state information. In this work, we have assumed that no cental controller or the destination has acquired CSI regarding all involved relay links. If that constraint is relaxed, or if the destination is able to estimate the channel towards the interfering relay, then optimum combining becomes an option.

\section{B. Selection Diversity Receiver (SR)}

When the receiver operates on the two, time-separated signal parts independently, the weighting vector $\mathbf{w}$ for each part is simplified to $\mathbf{w}_{\mathrm{SR}, 1}=\operatorname{diag}\left[\begin{array}{ll}1 & 0\end{array}\right] \mathbf{a}, \mathbf{w}_{\mathrm{SR}, 2}=\operatorname{diag}\left[\begin{array}{ll}0 & 1\end{array}\right] \mathbf{a}$.

Outage occurs when both decoding efforts fail and thus, conditional outage probability becomes:

$$
\begin{aligned}
& \operatorname{Pr}\left\{\text { out }\left|\mathcal{D}_{\ell},\right| \mathcal{D}_{\ell} \mid \geq 1, I=i\right\}_{\mathrm{SR}}= \\
& =\operatorname{Pr}\left(\frac{\zeta \mathcal{P} \gamma_{s d}}{(1-\zeta) \mathcal{P} \gamma_{i d}+N_{0}}<\Theta \bigcap \frac{(1-\zeta) \mathcal{P} \gamma_{b d}}{\zeta \mathcal{P} \gamma_{s d}+N_{0}}<\Theta\right) \\
& =\operatorname{Pr}\left(\frac{1-\zeta}{\zeta \Theta} \gamma_{b d}-\frac{N_{0}}{\zeta \mathcal{P}}<\gamma_{s d}<\Theta \frac{1-\zeta}{\zeta} \gamma_{i d}+\frac{\Theta N_{0}}{\zeta P}\right) \\
& =\mathbb{E}_{\gamma_{i d}}\left\{\mathbb{E}_{\gamma_{b d} \mid \gamma_{i d}}\left\{g\left(\gamma_{i d}, \gamma_{b d}\right)\right\}\right\}, \\
& g\left(\gamma_{i d}, \gamma_{b d}\right)=u\left(\Theta \frac{1-\zeta}{\zeta} \gamma_{i d}+\frac{(\Theta+1) N_{0}}{\zeta P}-\frac{1-\zeta}{\zeta \Theta} \gamma_{b d}\right) \times \\
& \times\left\{F_{s d}\left(\frac{\Theta(1-\zeta)}{\zeta} \gamma_{i d}+\frac{\Theta N_{0}}{\zeta P}\right)\right. \\
& \left.-F_{s d}\left(\frac{1-\zeta}{\zeta \Theta} \gamma_{b d}-\frac{N_{0}}{\zeta \mathcal{P}}\right) u\left(\gamma_{b d}-\nu_{0}\right)\right\},
\end{aligned}
$$


with $u(x)$ the unitary step function $(u(x)=1, x \geq 0$ and $u(x)=0$, elsewhere $)$ and

$$
F_{s d}(x) \triangleq \operatorname{Pr}\left(\gamma_{s d}<x\right)=1-e^{-\lambda_{s d} x}, \nu_{0}=\frac{\Theta N_{0}}{(1-\zeta) \mathcal{P}} .
$$

We note again that under the coherence time duration assumption of this work, $\gamma_{b d}$ is independent of $\gamma_{i d}$ (relay $i$ is the interfering relay).

Using Theorem 1 at the appendix, the p.d.f. of $\gamma_{b d}=$ $\max \left\{\gamma_{k d}\right\}, k \in \mathcal{D}_{\ell} \subseteq \mathcal{S}_{\mathrm{R}}^{i},\left|\mathcal{D}_{\ell}\right| \geq 1$ becomes:

$$
p_{\gamma_{b d}}(\gamma)=\sum_{m=1}^{\ell} \sum_{\substack{\mathcal{S}_{m} \subseteq \mathcal{D}_{\ell} \\ \mathcal{S}_{m} \mid=m}}(-1)^{m+1}\left(\sum_{\nu \in \mathcal{S}_{m}} \lambda_{\nu d}\right) e^{-\gamma \sum_{\nu \in \mathcal{S}_{m}} \lambda_{\nu d}}
$$

Exploiting eq. (20) in eq. (17) and the fact that

$$
\begin{aligned}
\sum_{m=1}^{\ell} \sum_{\substack{\mathcal{S}_{m} \subseteq \mathcal{D}_{\ell} \\
\left|\mathcal{S}_{m}\right|=m}}(-1)^{m+1}=-\sum_{m=1}^{\ell}\left(\begin{array}{c}
\ell \\
m
\end{array}\right)(-1)^{m} & \\
= & -\left(-1+\sum_{m=0}^{\ell}\left(\begin{array}{c}
\ell \\
m
\end{array}\right)(-1)^{m}\right)=1,
\end{aligned}
$$

the conditional to interfering relay outage probability for $\left|\mathcal{D}_{\ell}\right| \geq 1$, after manipulations becomes:

$$
\begin{aligned}
& \operatorname{Pr}\left\{\text { out }\left|\mathcal{D}_{\ell},\right| \mathcal{D}_{\ell} \mid \geq 1, I=i\right\}_{\mathrm{SR}} \\
& =1-e^{-\lambda_{s d} \frac{\Theta N_{0}}{\zeta P}}\left[1+\Theta \frac{1-\zeta}{\zeta} \frac{\lambda_{s d}}{\lambda_{i d}}\right]^{-1} \\
& -\sum_{m=1}^{\ell} \sum_{\substack{\mathcal{S}_{m} \subseteq \mathcal{D}_{\ell} \\
\left|\mathcal{S}_{m}\right|=m}}(-1)^{m+1} \frac{\exp \left\{-\nu_{0} \sum_{\nu \in \mathcal{S}_{m}} \lambda_{\nu d}\right\}}{1+\Theta \frac{\zeta}{1-\zeta} \frac{1}{\lambda_{s d}} \sum_{\nu \in \mathcal{S}_{m}} \lambda_{\nu d}}+ \\
& +\sum_{m=1}^{\ell} \sum_{\substack{\mathcal{S}_{m} \subseteq \mathcal{D}_{\ell} \\
\left|\mathcal{S}_{m}\right|=m}}\left\{(-1)^{m+1} \frac{e^{-\lambda_{s d} \frac{\Theta N_{0}}{\zeta \mathcal{P}}}}{1+\Theta \frac{\zeta}{1-\zeta} \frac{1}{\lambda_{s d}} \sum_{\nu \in \mathcal{S}_{m}} \lambda_{\nu d}} \times\right. \\
& \left.\quad \times \frac{\exp \left\{-\frac{\Theta(\Theta+1) N_{0}}{(1-\zeta) \mathcal{P}} \sum_{\nu \in \mathcal{S}_{m}} \lambda_{\nu d}\right\}}{1+\Theta \frac{1-\zeta}{\zeta} \frac{\lambda_{s d}}{\lambda_{i d}}+\frac{\Theta^{2}}{\lambda_{i d}} \sum_{\nu \in \mathcal{S}_{m}} \lambda_{\nu d}}\right\} .
\end{aligned}
$$

For partial connectivity scenarios where there is no direct link between source and destination $\left(\lambda_{s d} \rightarrow+\infty\right)$, eq. (22) becomes:

$$
\begin{aligned}
& \operatorname{Pr}\left\{\text { out }\left|\mathcal{D}_{\ell},\right| \mathcal{D}_{\ell} \mid \geq 1, I=i, \lambda_{s d}^{-1} \rightarrow 0\right\}_{\mathrm{SR}} \\
& =\sum_{m=0}^{\ell} \sum_{\substack{\mathcal{S}_{m} \subseteq \mathcal{D}_{\ell} \\
\left|\mathcal{S}_{m}\right|=m}}(-1)^{m} \exp \left\{-\nu_{0} \sum_{\nu \in \mathcal{S}_{m}} \lambda_{\nu d}\right\} \\
& \stackrel{(a)}{=} \prod_{m \in \mathcal{D}_{\ell}}\left(1-e^{-\nu_{0} \lambda_{m d}}\right)=\prod_{m \in \mathcal{D}_{\ell}}\left(1-e^{-\lambda_{m d} \frac{\Theta N_{0}}{(1-\zeta) \mathcal{P}}}\right),
\end{aligned}
$$

where we have used in step $(a)$ the multinomial identity of eq. (33) at the appendix. The last conditional outage probability (with $\gamma_{s d} \rightarrow 0$ ), depends on thermal noise only, as expected.

\section{A Note on Interference-Limited ProACtive SENSING}

In an alternative approach, the most appropriate relay could be selected proactively, before the source message transmission. In that way, all non-selected relays could avoid listening to the source, and thus, save precious battery energy. Denoting

$$
\begin{aligned}
A^{(i)} & =\frac{\zeta \mathcal{P} \gamma_{s d}}{(1-\zeta) \mathcal{P} \gamma_{i d}+N_{0}}, B_{1}^{(i, j)}=\frac{\zeta \mathcal{P} \gamma_{s j}}{(1-\zeta) \mathcal{P} \gamma_{i j}+N_{0}}, \\
B_{2}^{(j)} & =\frac{(1-\zeta) \mathcal{P} \gamma_{j d}}{\zeta \mathcal{P} \gamma_{s d}+N_{0}},
\end{aligned}
$$

the proactive outage probability, with selection receiver at the destination and conditioned on interfering relay $i$, becomes:

$$
\begin{aligned}
& \stackrel{\text { proactive }}{\operatorname{Pr}}\{\text { out } \mid I=i\} \\
& =\operatorname{Pr}\left\{A^{(i)}<\Theta \bigcap\left(B_{1}^{(i, j)}<\Theta \bigcup B_{2}^{(j)}<\Theta\right)\right\} \\
& =\operatorname{Pr}\left\{\max \left\{A^{(i)}, B_{1}^{(i, j)}\right\}<\Theta \bigcup \max \left\{A^{(i)}, B_{2}^{(j)}\right\}<\Theta\right\} \\
& =\operatorname{Pr}\{\underbrace{\min \left\{\max \left\{A^{(i)}, B_{1}^{(i, j)}\right\}, \max \left\{A^{(i)}, B_{2}^{(j)}\right\}\right.}_{W_{j, i}}<\Theta\} \\
& \equiv \operatorname{Pr}\left\{W_{j, i}<\Theta\right\} .
\end{aligned}
$$

The best relay should minimize outage probability and thus maximize the following expression:

$$
W_{b, i} \geq W_{j, i}, \forall j \in \mathcal{S}_{\mathrm{R}}^{i}
$$

However, the relays cannot estimate the channel between source and destination, which is needed in $A^{(i)}, B_{2}^{(j)}$ above, unless the source or the destination provides them with such information. In that case however, implementation of proactive selection becomes non-distributed. An alternative, sub-optimal (in terms of outage probability) but practical (in terms of distributed implementation) proactive selection rule ignores the link between source and destination:

$$
\widehat{W}_{j, i}=\min \left\{B_{1}^{(i, j)}, B_{3}^{(j)}\right\}, B_{3}^{(j)}=\frac{(1-\zeta) \mathcal{P} \gamma_{j d}}{N_{0}} .
$$

We restrict analysis on reactive sensing and note that the relay selection rule of eq. (2) is exactly the same as in strictly noise-limited environments [2]. We also remark that other opportunistic relay selection rules are possible in the presence of both interference and thermal noise, when additional CSI is available (e.g. information regarding the interfering relay link). Hopefully, this work will spark interest in relevant research that aims to optimize performance in the presence of interference, without excessive overheads in channel state information acquisition and network coordination.

\section{NumericAl RESUlts}

Numerical results are provided for the two kinds of receivers, assuming a weak link (and thus, high corresponding $\lambda$ parameter) between source and destinations, with $\lambda_{s d}=$ $1 / 0.05$. The number of relays $K$ is set to $K=3$ or $K=4$ relays with $\zeta=0.5, R=0.5 \mathrm{bits} / \mathrm{sec} / \mathrm{Hz}$ and $\lambda_{s j}=1 / 1.6$, $\lambda_{j d}=1 / 1.5 \forall j \in \mathcal{S}_{\mathrm{R}}, \lambda_{j i}=1 / 0.05 \forall j \in \mathcal{S}_{\mathrm{R}}^{i}$. Such parameters correspond to a small number of relays that are "isolated" from each other, while providing "good" communication links 


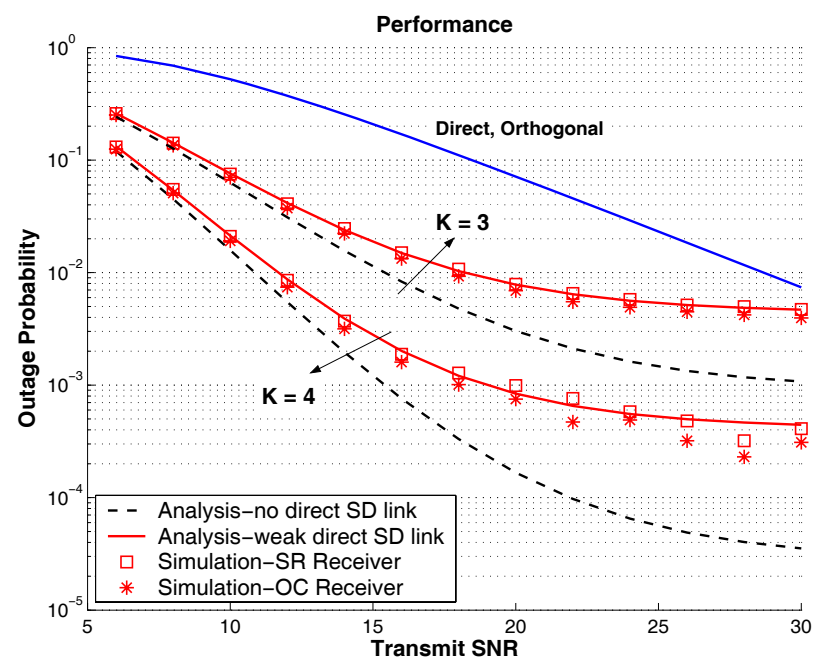

Fig. 4. Acceptable performance of interference-aware OR with $K$ relays is feasible. Two scenarios for source-destination (SD) links are depicted. Performance of noise-limited (orthogonal), non-cooperative communication is also depicted.

(on average) towards source and destinations. For example, assuming transmit signal-to-noise ratio $\mathrm{SNR}=\mathcal{P} / N_{0}$ ranging from 0 to $30 \mathrm{~dB}$, the above parameters correspond to average received signal-to-noise ratio approximately from -1 to 29 $\mathrm{dB}$ at each relay or each destination.

Under such parameters, the upper and lower bound of outage probability coincide (Eq. (6)). Fig. 4 demonstrates that simulation results match analysis for SR performance. Moreover, OC performance is improved by approx. $3 \mathrm{~dB}$ compared to SR, at the expense of additional required CSI. For both receivers, performance reaches an outage probability plateau, which is due to the interference-limited nature of the protocol. However, that outage probability plateau decreases with increasing number of utilized relays, even though a single relay transmits at each phase. That is due to the opportunistic nature of the protocol, based on reactive sensing and the relay selection rule of Eq. (2).

Fig. 4 also includes the case of no-link between source and destination (intermittent line), which provides smaller outage probability than the scenario with existing (but weak) direct links; that is because interference at the destination is eliminated during phase II of each epoch. The case of direct, noise-limited communication is also depicted, which tests $\operatorname{Pr}\left((1+\mathrm{RSO}) \log _{2}\left(1+\gamma_{s d} \frac{\mathcal{P}}{N_{0}}\right)<R\right)$. The parameter RSO denotes the relay selection overhead, i.e. the percentage of channel coherence time spent for relay selection in the cooperative case. This is added for fairness to direct communication and is set to RSO $=10 \%$.

Table I provides the connection between outage probability and bit-error-rate (BER), assuming independent errors across

TABLE I

Outage Probability vs BER

\begin{tabular}{c|cc} 
Outage Probability & BER for $T_{c}=125 \mathrm{msec}, R_{b}=11 \mathrm{Mbps}$ \\
\hline \hline $\operatorname{Pr}\{$ out $\}=10^{-2}$ & $\approx 10^{-8}$ \\
$\operatorname{Pr}\{$ out $\}=10^{-3}$ & $\approx 10^{-9}$
\end{tabular}

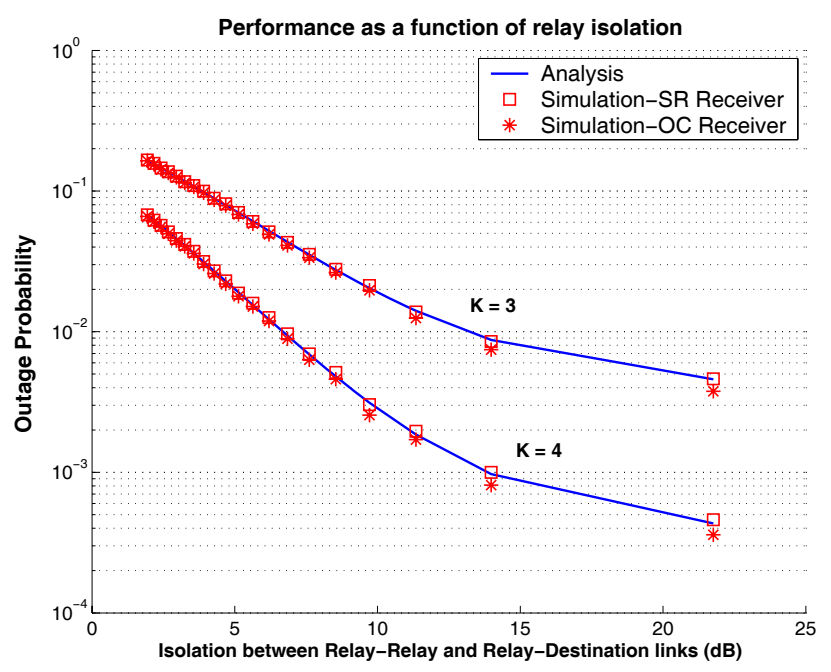

Fig. 5. Performance of interference-aware OR with $K$ relays as a function of relay isolation $\mathbb{E}\left\{\gamma_{j d}\right\} / \mathbb{E}\left\{\gamma_{j i}\right\}$ (in $\mathrm{dB}$ ), for transmit $\mathrm{SNR}=20 \mathrm{~dB}$.

$N_{b}$ bits: $(1-\mathrm{BER})^{N_{b}} \simeq 1-\operatorname{Pr}$ \{out\}, with $N_{b}=\frac{T_{c}}{2} R_{b}$, where $R_{b}$ is the transmission rate in bps. For slow mobility (walking speeds) and channel coherence time on the order of $125 \mathrm{msec}$, the observed outage probability plateau of Fig. 4 could provide for BER less than $10^{-8}$. The latter is required in reliability-demanding applications as in video transmission. It is also noted that $802.11 \mathrm{~b}$ operates on maximum $R_{b}=11$ Mbps (@22 MHz bandwidth). For larger $T_{c}$, BER becomes even smaller. Thus, interference-limited OR could assist demanding applications.

Fig. 5 sets transmit $\mathrm{SNR}=\mathcal{P} / N_{0}=20 \mathrm{~dB}$ and varies $\mathbb{E}\left\{\gamma_{j i}\right\}$, investigating the required amount of isolation between relays (while keeping the rest of parameters same as in Fig. 4 with weak source-destination link). Specifically, performance is tested against various values of relay isolation $\mathbb{E}\left\{\gamma_{j d}\right\} / \mathbb{E}\left\{\gamma_{j i}\right\}$ (in $\mathrm{dB}$ ). It is shown that relevantly small value of $10 \mathrm{~dB}$ isolation is required for $K=4$ relays and outage probability well below $1 \%$. It is noted that for $11 \mathrm{Mbps}$ transmission rate and channel coherence time on the order of $125 \mathrm{msec}$ (e.g. walking speed), outage probability of $1 \%$ corresponds to bit-error-rate (BER) less than $10^{-8}$, assuming binary modulation and independent errors across bits.

Practical ways to engineer such isolation among relays could facilitate directive antennas at the relays, including lowcomplexity beam-switched Butler matrix antennas. The latter provide side lobe level attenuation (SLL) (Fig. 3) well below $-10 \mathrm{~dB}$ compared to the main lobe direction (e.g. see [8]) and thus, could potentially engineer the required minimum $10-\mathrm{dB}$ "isolation" between relays. The interested reader could also refer to [8]-[9] for additional information regarding practical designs of low-complexity, beam-switching antennas.

\section{CONCLUSION}

Under the main assumption of this work, it was shown that information can be relayed without delay, while harvesting benefits of cooperative diversity, even at the presence of interference. The participating relays are required to offer strong paths towards source and destination, while at the same 
time they are as "isolated" as possible from each other. From that perspective, the notion of relay "usefulness" is redefined in both noise and interference-limited environments, under opportunistic relaying. In sharp contrast to prior art, no interrelay CSI or communication was assumed, no network coding was needed, while low-complexity receivers at each destination were employed. Hopefully, this work may potentially spark further interest in efficient opportunistic relay selection algorithms for interference-limited, slow-fading, dense, wireless access networks.

\section{APPENDIX}

Theorem 1 (Max Distribution): Let $Y_{n}, n=1,2, \ldots, M$, be $M$ statistically independent and not necessarily identically distributed (i.n.i.d.) exponential r.v.'s, each with parameter $\lambda_{n}=1 / \mathbb{E}\left\{Y_{n}\right\}$. Then, the p.d.f. of $X=\max \left\{Y_{1}, Y_{2}, \ldots, Y_{M}\right\}$ for $x \geq 0$ is given by:

$$
\begin{aligned}
& p_{X}(x)= \\
& =\sum_{m=1}^{M} \sum_{\substack{\mathcal{S}_{m} \subseteq\{1,2, \ldots, M\} \\
\left|\mathcal{S}_{m}\right|=m}}(-1)^{m+1}\left(\sum_{j \in \mathcal{S}_{m}} \lambda_{j}\right) e^{-x \sum_{j \in \mathcal{S}_{m}} \lambda_{j}}
\end{aligned}
$$

Proof: Since $Y_{1}, Y_{2}, \ldots, Y_{M}$ are statistically independent and exponentially distributed, the c.d.f. of $X$ can be readily calculated:

$$
\begin{aligned}
F_{X}(x) & =\operatorname{Pr}\{X \leq x\}=\prod_{\beta=1}^{M}\left(1-e^{-\lambda_{i} x}\right) \\
& \stackrel{(*)}{=} 1+\sum_{m=1}^{M} \sum_{\substack{\mathcal{S}_{m} \subseteq\{1,2, \ldots, M\} \\
\left|\mathcal{S}_{m}\right|=m}}(-1)^{m} e^{-x \sum_{j \in \mathcal{S}_{m}} \lambda_{j}}
\end{aligned}
$$

where we used in step $(*)$ the multinomial identity:

$$
\prod_{i=1}^{K}\left(1-a_{i}\right)=1+\sum_{\ell=1}^{K} \sum_{\substack{\mathcal{S}_{\ell} \subseteq\{1,2, \ldots, K\} \\\left|\mathcal{S}_{\ell}\right|=\ell}}(-1)^{\ell} \prod_{j \in \mathcal{S}_{\ell}} a_{j} .
$$

Thus, the p.d.f. of $X$ becomes:

$$
\begin{aligned}
p_{X}(x) & =\sum_{i=1}^{M} \lambda_{i} e^{-\lambda_{i} x} \prod_{\substack{j=1 \\
j \neq i}}^{M}\left(1-e^{\lambda_{j} x}\right) \\
& =\sum_{m=1}^{M} \sum_{\substack{\mathcal{S}_{m} \subseteq\{1,2, \ldots, M\} \\
\left|\mathcal{S}_{m}\right|=m}}(-1)^{m} \frac{d}{d x}\left(e^{-x \sum_{j \in \mathcal{S}_{m}} \lambda_{j}}\right)
\end{aligned}
$$

providing the result of eq. (30).

\section{REFERENCES}

[1] J. N. Laneman and G. W. Wornell, "Distributed space-time coded protocols for exploiting cooperative diversity in wireless networks," IEEE Trans. Inf. Theory, vol. 59, pp. 2415-2525, Oct. 2003.

[2] A. Bletsas, H. Shin, and M. Z. Win, "Cooperative communications with outage-optimal opportunistic relaying," IEEE Trans. Wireless Commun., vol. 6, no. 9, pp. 3450-3460, Sept. 2007.

[3] N. Fawaz, D. Gesbert, and M. Debbah, "When network coding and dirty paper coding meet in a cooperative ad hoc network," IEEE Trans. Wireless Commun., vol. 7, no. 5, pp. 1862-1867, May 2008.

[4] K. Jones and L. Liu, "What where Wi: An analysis of millions of WiFi access points," in Proc. IEEE Int. Conf. Portable Inf. Devices, Orlando, FL, Mar. 2007.

[5] A. Bletsas, A. Khisti, D. P. Reed, and A. Lippman, "A simple cooperative diversity method based on network path selection," IEEE J. Sel. Areas Commun., special issue on $4 \mathrm{G}$ wireless systems, vol. 24, no. 9, pp. 659672, Mar. 2006.

[6] Q. T. Zhang and X. W. Cui, "Outage probability for optimum combining of arbitrarily faded signals in the presence of correlated Rayleigh interferers," IEEE Trans. Veh. Technol., vol. 53, pp. 1043-1051, July 2004.

[7] J. M. Romero-Jerez and A. J. Goldsmith, "Receive antenna array strategies in fading and interference: an outage probability comparison," IEEE Trans. Wireless Commun., vol. 7, pp. 920-932, Mar. 2008.

[8] E. Siachalou, E. Vafiadis, S. Goudos, T. Samaras, C. Koukourlis, and S. Panas, "On the design of switched-beam wideband base stations," IEEE Antennas Propagat. Mag., vol. 46, pp. 158-167, Feb. 2004.

[9] E. Vaitsopoulos, A. Bletsas, and J. N. Sahalos, "On the RFID design with passive tags and a butler matrix reader," in Proc. IEEE 13th Biennial Conf. Electromagnetic Field Computation, May 2008. 